Original Research

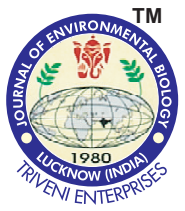

DOI : http://doi.org/10.22438/jeb/39/4/MRN-590

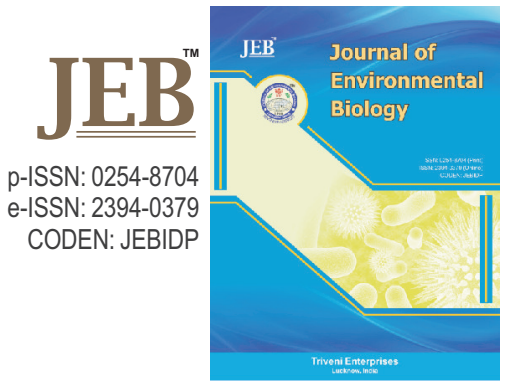

\title{
Towards enhanced low temperature stress tolerance in tomato : An approach
}

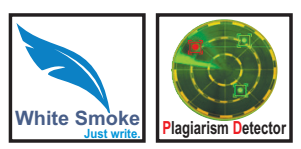

Authors Info

Y.K. Meena ${ }^{1 *}$, D.S. Khurana', Nirmaljit Kaur and Kulbir Singh ${ }^{1}$

${ }^{1}$ Department of Vegetable Science, Punjab Agricultural University, Ludhiana -141 004, India

${ }^{2}$ Department of Botany, Punjab Agricultural University, Ludhiana -141 004, India

*Corresponding Author Email : yogendra-coavs@pau.edu

Key words

Low temperature

Phenolic compounds

Physiological traits

Stress tolerance

Tomato yield

\section{Publication Info}

Paper received : 13.02.2017

Revised received: 12.06 .2017

Re-revised received : 24.12.2017

Accepted: 11.01.2018

\section{Abstract}

Aim : The aim of the present study was to find suitable concentration of phenolic compounds to ameliorate the effect of low temperature stress in tomato under open field conditions during winter season.

Methodology : The average minimum temperatures were below $10^{\circ} \mathrm{C}$ from mid December to mid February during both the years. The experimental plants were given foliar application twice, first 15 days after transplanting followed by another spray after a fortnight of five different phenolic compounds viz., salicylic acid, sulfo salicylic acid, benzoic acid, methyl salicylic acid and acetyl salicylic acid with different concentrations of $0.1 \mathrm{mM}, 0.5 \mathrm{mM}$ and $1.0 \mathrm{mM}$ in order to evaluate their effect on morphological, physiological, yield and quality traits.

Results : The results obtained revealed that low temperature during winter season significantly reduced the growth and physiological traits (leaf are a index, specific leaf area, relative growth rate, net assimilation rate and relative water content), yield attributes (number of flower per cluster, number of fruits and fruit yield) and quality parameters (lycopene, $\beta$-carotene and $\alpha$-tocopherol) and increased specific leaf weight and electrolyte leakage. However, application of phenolic compounds significantly enhanced the growth, yield, quality and decreased electrolyte leakage and specific leaf weight under stress. Among all treatments, $\mathrm{SA}(1.0 \mathrm{mM})$ was found most effective to enhance low temperature stress tolerance in tomato.

Interpretation : Cold stress is one of the environmental cues that limits the crop production. Phenolic compounds play a significant role as signaling molecules in conferring cold stress tolerance in plants. It may thus be concluded that foliar application of salicylic acid (SA) (1.0 mM) enhanced low temperature stress tolerance in tomato.

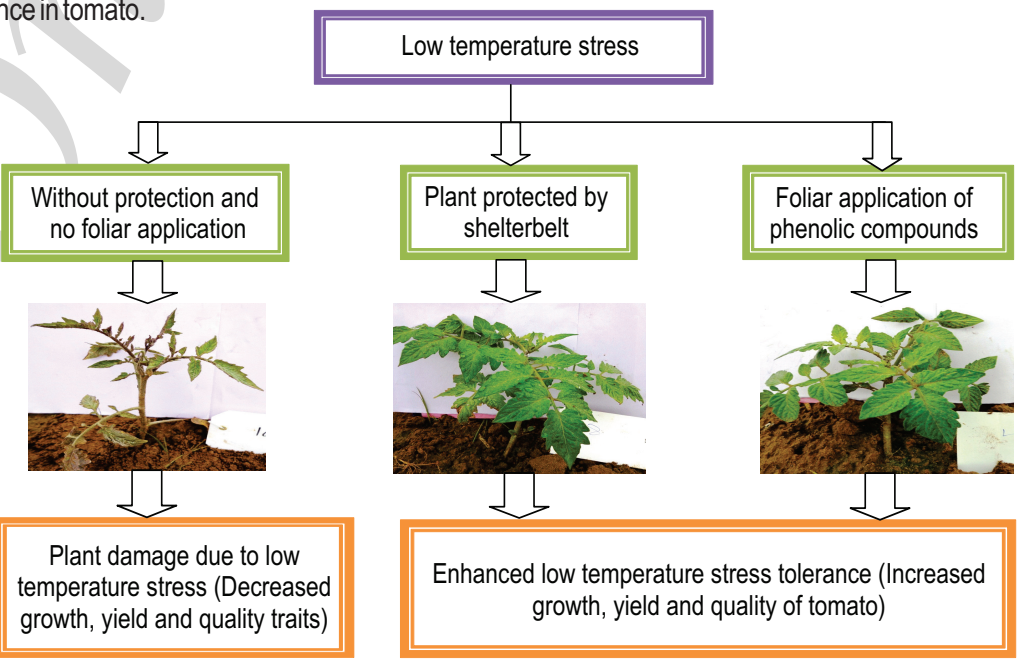




\section{Introduction}

Tomato (Solanum lycopersicum L.) is one of the most common and widely grown vegetable in the world, ranks second in importance after potato. It is grown throughout the world and is a rich source of lycopene, vitamins and minerals. Lycopene is the main compound responsible for the characteristic deep red colour of ripe tomato fruits and tomato products (Helyes et al., 2009). It is the key intermediate in the biosynthesis of important carotenoids, such as $\beta$-carotene and xanthophylls and may help in counteracting the harmful effects of substances called "free radicals" and different types of cancers. Tomato, one of the most important vegetable crops in the world encounters low and high temperature stress as two huge challenges for its production.

Improved agriculture is important to address global food security and environmental stress tolerant crops have provided promising solutions. Low and high temperature stress are becoming the major researchable issues for agricultural scientists worldwide due to the challenges imposed by changing climate (Shah et al., 2011). Abiotic stress can reduce growth, development, yield and quality which causes valuable crop loss in terms of productivity of tomato (Khan et al., 2015). Low temperature stress causes propound morphological and physiological changes that adversely affect plant growth, development and ultimately yield and quality (Sayyari et al., 2013; Khan et al., 2015). Tomato being an important summer vegetable crop of India is quite sensitive to cold stress and shows reduction in overall growth, yield and quality contributing parameters (Shi et al., 2012; Khan et al., 2015). Development of methods to enhance stress tolerance in plants is crucial and generates considerable attention. Low temperature stress adversely affects the reproductive phase leading to increased flower abortion and fruit set. Plants grown in cold stress have significantly reduced number of fruits and fruit yield per plant and the rate of reduction in fruit number has been reported to be in proportion to the level of cold stress in tomato (Khan et al., 2015). At harvest, fruits developed on the plants grown in low temperature stress possess significantly lower values for lycopene and $\beta$-carotene contents. This loss increased further with a decrease in the level of low temperature stress in tomato (Khan et al., 2015).

Phenolic compounds act as a vital signal molecule for modulating plant response to various abiotic stresses (Hayat etal., 2012). Phenolic compound induced alleviation of low temperature stress has been reported in tomato (Orabi et al., 2015), cucumber (Zhang et al., 2015) and watermelon (Sayyari et al., 2013). Application of phenolic compounds assist in improving the tolerance against low temperature stress in plants. Moreover, information on suitable concentration of phenolic compounds to mitigate low temperature stress in tomato under open field condition is meager. Besides this, field appraisals of exogenously applied phenolic compounds are also lacking. This study was meant to depict the role of foliar application of phenolic compounds on morphology, physiological biomarkers, yield and quality contributing traits of tomato plants at low temperature stress under open field conditions during winter season.

\section{Materials and Methods}

Plant material and stress conditions : Seeds of tomato (Solanum lycopersicum L.) variety Punjab Ratta were obtained from the Department of Vegetable Science, Punjab Agricultural University, Ludhiana, India. Seeds were sown in nursery plug tray and covered with polyethylene to protect from low temperature. Twenty eight days after sowing, seedlings were transplanted on first date of December 2014 and 2015 under open field conditions; the average minimum and maximum temperatures of two years are shown in Fig. 1. The cultural practices used were as per Punjab Agricultural University recommended practices. Foliar applications of phenolic compound with different concentration $(0.1,0.5$ and $1.0 \mathrm{mM})$ were given 15 and 30 days after transplanting (DAT). In one treatment, plants were protected by shelterbelt (Covered by polyethylene, as per, recommended by Punjab Agricultural University) and in control, plants were given foliar spray of distilled water. The experiment was designed in a complete randomized block design with three replications. In each plot, 40 plants were sown and 5 plants were randomly selected for growth and physiological attributes at 30,60 and 90 days after transplanting and remaining for yield and quality parameters. Growth parameters, leaf area index, specific leaf area and specific leaf weight recorded as per calculation formula described by Fahraji et al. (2014). Physiological attributes viz., relative leaf water content from the leaves of plants was recorded as per method described by Kaya et al. (2003), membrane stability was calculated by the method given by Shi et al. (2006). Quality parameters were estimated by the following methods lycopene content (Sadasivam and Manickam, 1992), $\beta$ - carotene content (Watanabe et al., 1991) and a-tocopherol content (Asthir et al., 2009).

Statistical analysis : Data were analyzed using STAR 2.0.1 software developed by International Rice Research Institute. The mean comparisons were performed by Duncan's multiple range testat $p<0.05$.

\section{Results and Discussion}

Low temperature stress adversely affects plant growth and development. Cold stress significantly caused reduction in most of the growth biomarkers viz., leaf area index $(56.09 \%$, $56.81 \%$ and $61.11 \%)$, specific leaf area $(29.41 \%, 27.77 \%$ and $28.51 \%)$, relative growth rate $(8.85 \%, 9.85 \%$ and $8.54 \%)$, net assimilation rate $(42.18 \%, 47.05 \%$ and $64.52 \%)$ and enhanced specific leaf weight $(41.26 \%, 81.12 \%$ and $42.25 \%)$ at 30,60 and 90 days after transplanting during both the years as compared to plants protected by shelterbelt (Table 1 and 2). The degree of damage caused due to low temperature stress during two years in tomato was different at different growth stages. The minimum growth biomarkers were observed in non treated plants during both years of study. Low temperature stress affects crop yield by 


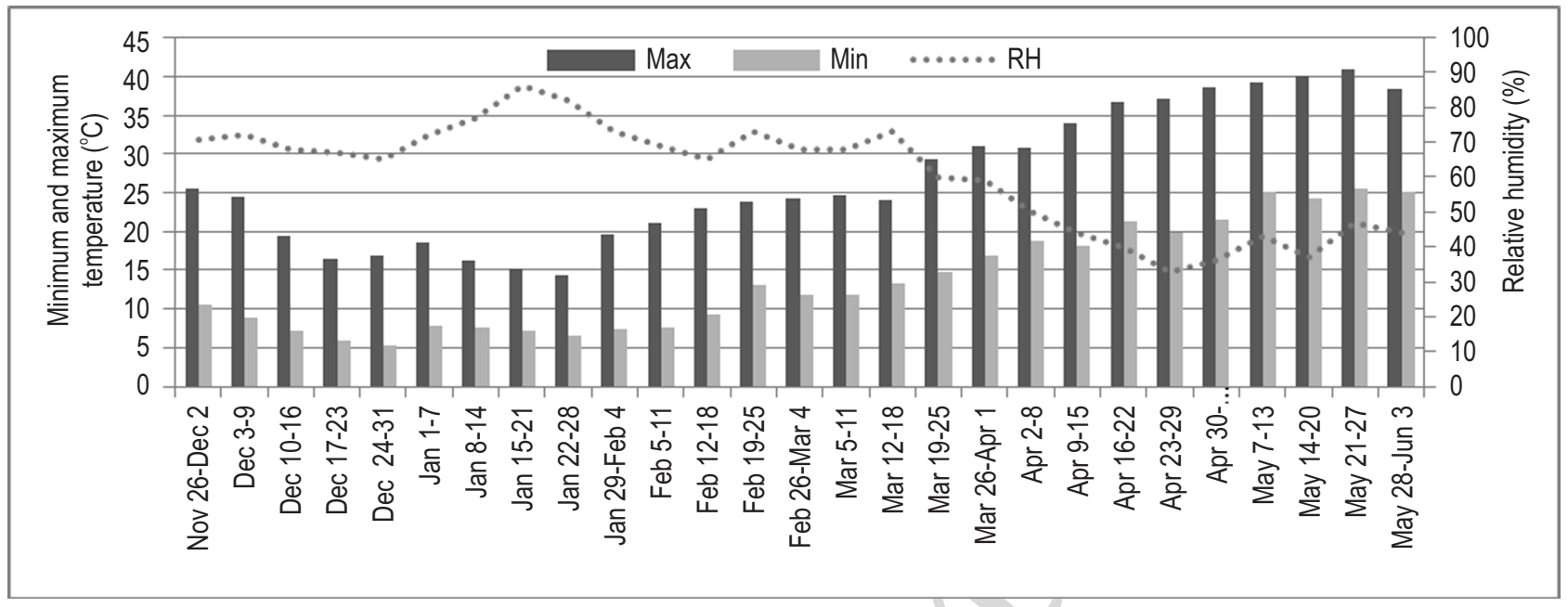

Fig. 1 : Average means of minimum, maximum temperature $\left({ }^{\circ} \mathrm{C}\right)$ and relative humidity $(\%)$ in Punjab during 2014-15 and 2015-16

reducing plant growth, with negative and irregular effects on biomass accumulation (Khan et al., 2015). Low temperature caused tissue discoloration and increased water loss, as a result of suppressed expression of genes which is generally active at normal temperatures (Saltveit and Morris, 1990). In addition to this, Lukatkin et al. (2012) reported a fast decresase in the number of dividing cells during low temperature; decreased mitotic index in apices and basal part of young leaves. Cell growth was inhibited which caused considerable changes in plant organs (Strauss etal., 2007).

Table 1 : Effects of phenolic compounds on LAI, SLA and SLW in tomato under low temperature stress condition in Punjab during 2014-16

\begin{tabular}{|c|c|c|c|c|c|c|c|c|c|}
\hline \multirow[t]{2}{*}{ Treatments } & \multicolumn{3}{|c|}{ Leaf area index (LAl) } & \multicolumn{3}{|c|}{$\begin{array}{l}\text { Specific leaf area (SLA) } \\
\left(\mathrm{cm}^{2} \mathrm{mg}^{-1} \text { plant }\right.\end{array}$} & \multicolumn{3}{|c|}{$\begin{array}{l}\text { Specific leaf weight (SLW) } \\
\left(\mathrm{mg} \mathrm{cm}^{2} \text { plant } \mathrm{C}^{-1}\right)\end{array}$} \\
\hline & 30DAT & 60DAT & 90DAT & 30DAT & 60DAT & 90DAT & 30DAT & 60DAT & 90DAT \\
\hline \multicolumn{10}{|l|}{ Salicylic acid } \\
\hline $0.1 \mathrm{~m} \mathrm{M}$ & $0.26^{\text {ghi }}$ & $2.16^{\mathrm{ef}}$ & $4.52^{\mathrm{e}}$ & $0.15^{\text {cde }}$ & $0.16^{\text {cde }}$ & $0.228^{\mathrm{e}}$ & $6.54^{\mathrm{bcd}}$ & $6.71^{\mathrm{cd}}$ & $4.53^{\mathrm{bcd}}$ \\
\hline $0.5 \mathrm{~m} \mathrm{M}$ & $0.31^{e-i}$ & $2.67^{\mathrm{bcd}}$ & $5.17^{\text {cde }}$ & $0.16^{a-e}$ & $0.17^{\mathrm{a}-\mathrm{e}}$ & $0.240^{\text {bcde }}$ & $6.23^{b-9}$ & $6.14^{\mathrm{d}-\mathrm{i}}$ & $4.24^{c-h}$ \\
\hline $1.0 \mathrm{~m} \mathrm{M}$ & $0.40^{\mathrm{ab}}$ & $3.02^{\mathrm{ab}}$ & $6.36^{\mathrm{ab}}$ & $0.17^{\mathrm{a}}$ & $0.18^{\mathrm{a}}$ & $0.257^{\mathrm{ab}}$ & $5.84^{\text {gh }}$ & $5.40^{\mathrm{hi}}$ & $3.89^{\mathrm{hi}}$ \\
\hline \multicolumn{10}{|c|}{ Methyl salicylic acid } \\
\hline $0.1 \mathrm{~m} \mathrm{M}$ & $0.26^{i}$ & $1.79^{9}$ & $4.24^{\mathrm{e}}$ & $0.15^{\mathrm{e}}$ & $0.15^{\mathrm{e}}$ & $0.224^{\mathrm{e}}$ & $6.72^{\mathrm{b}}$ & $7.31^{\mathrm{b}}$ & $4.67^{b}$ \\
\hline $0.5 \mathrm{~m} \mathrm{M}$ & $0.31^{e-i}$ & $2.41^{\text {cde }}$ & $4.96^{\text {cde }}$ & $0.16^{a-e}$ & $0.16^{a-e}$ & $0.235^{\text {bcde }}$ & $6.40^{b-f}$ & $6.47^{\text {defg }}$ & $4.38^{b-f}$ \\
\hline $1.0 \mathrm{~m} \mathrm{M}$ & $0.33^{\text {def }}$ & $2.71^{\mathrm{abcd}}$ & $5.75^{\mathrm{abcd}}$ & $0.16^{a-e}$ & $0.17^{\mathrm{a}-\mathrm{e}}$ & $0.245^{a-e}$ & $6.16^{c-h}$ & $6.10^{\mathrm{e}-\mathrm{i}}$ & $4.16^{\mathrm{d}-\mathrm{i}}$ \\
\hline \multicolumn{10}{|c|}{ Sulfo salicylic acid } \\
\hline $0.1 \mathrm{~m} \mathrm{M}$ & $0.26^{i}$ & $2.18^{e f}$ & $4.46^{e}$ & $0.15^{\mathrm{de}}$ & $0.16^{\text {cde }}$ & $0.229^{\text {de }}$ & $6.54^{\mathrm{bcd}}$ & $6.67^{\text {cde }}$ & $4.49^{\mathrm{bcd}}$ \\
\hline $0.5 \mathrm{~m} \mathrm{M}$ & $0.32^{\text {efg }}$ & $2.71^{\text {abcd }}$ & $5.28^{\text {bcde }}$ & $0.16^{a-e}$ & $0.17^{\mathrm{a}-\mathrm{e}}$ & $0.239^{\text {bcde }}$ & $6.27^{b-h}$ & $6.23^{\mathrm{d}-\mathrm{h}}$ & $4.26^{c-h}$ \\
\hline $1.0 \mathrm{~m} \mathrm{M}$ & $0.37^{\mathrm{abcd}}$ & $2.92^{\mathrm{ab}}$ & $5.88^{\mathrm{abcd}}$ & $0.17^{\mathrm{ab}}$ & $0.17^{\mathrm{abc}}$ & $0.251^{\mathrm{abcd}}$ & $5.94^{\text {foh }}$ & $5.79^{\mathrm{hi}}$ & $4.02^{\text {fghi }}$ \\
\hline \multicolumn{10}{|l|}{ Benzoicacid } \\
\hline $0.1 \mathrm{~m} \mathrm{M}$ & $0.26^{\mathrm{hi}}$ & $1.99^{\mathrm{fg}}$ & $4.30^{\circ}$ & $0.15^{\mathrm{e}}$ & $0.16^{\mathrm{de}}$ & $0.226^{\mathrm{e}}$ & $6.61^{\mathrm{bc}}$ & $7.07^{\mathrm{bc}}$ & $4.60^{\mathrm{bc}}$ \\
\hline $0.5 \mathrm{~m} \mathrm{M}$ & $0.32^{\text {efgh }}$ & $2.51^{\mathrm{cde}}$ & $4.98^{\text {cde }}$ & $0.16^{a-e}$ & $0.16^{a-e}$ & $0.237^{\text {bcde }}$ & $6.36^{b-g}$ & $6.39^{\text {defg }}$ & $4.31^{\mathrm{b}-\mathrm{g}}$ \\
\hline $1.0 \mathrm{~m} \mathrm{M}$ & $0.34^{\text {cde }}$ & $2.75^{\mathrm{abcd}}$ & $4.83^{\mathrm{de}}$ & $0.16^{\mathrm{abc}}$ & $0.17^{\mathrm{a}-\mathrm{d}}$ & $0.241^{a-e}$ & $6.00^{\text {efgh }}$ & $6.03^{\text {fghi }}$ & $4.15^{\mathrm{e}-\mathrm{i}}$ \\
\hline \multicolumn{10}{|c|}{ Acetyl salicylic acid } \\
\hline $0.1 \mathrm{~m} \mathrm{M}$ & $0.27^{\text {fghi }}$ & $2.36^{\text {def }}$ & $4.57^{e}$ & $0.15^{\text {bcde }}$ & $0.16^{\text {bcde }}$ & $0.232^{\text {cde }}$ & $6.48^{\text {bcde }}$ & $6.60^{\text {cdef }}$ & $4.43^{\text {bcde }}$ \\
\hline $0.5 \mathrm{~m} \mathrm{M}$ & $0.39^{\mathrm{abc}}$ & $2.95^{\mathrm{ab}}$ & $6.05^{\mathrm{abc}}$ & $0.17^{\mathrm{a}}$ & $0.18^{\mathrm{ab}}$ & $0.254^{\mathrm{abc}}$ & $5.88^{\text {gh }}$ & $5.77^{\mathrm{hi}}$ & $3.96^{\text {ghi }}$ \\
\hline $1.0 \mathrm{~m} \mathrm{M}$ & $0.35^{\text {bcde }}$ & $2.80^{\mathrm{abc}}$ & $5.31^{\text {bcde }}$ & $0.16^{\mathrm{abcd}}$ & $0.17^{\mathrm{abcd}}$ & $0.247^{\mathrm{a}-\mathrm{e}}$ & $6.05^{\mathrm{d}-\mathrm{h}}$ & $5.90^{\text {ghi }}$ & $4.10^{\mathrm{e}-\mathrm{i}}$ \\
\hline Shelterbelt & $0.41^{a}$ & $3.08^{\mathrm{a}}$ & $6.61^{\mathrm{a}}$ & $0.17^{\mathrm{a}}$ & $0.18^{\mathrm{a}}$ & $0.263^{\mathrm{a}}$ & $5.84^{\text {gh }}$ & $5.60^{i}$ & $3.81^{i}$ \\
\hline Control (DW) & $0.18^{\mathrm{j}}$ & $1.33^{h}$ & $2.57^{\dagger}$ & $0.12^{f}$ & $0.13 f$ & $0.188^{f}$ & $8.52^{\mathrm{a}}$ & $10.15^{\mathrm{a}}$ & $5.42^{\mathrm{a}}$ \\
\hline
\end{tabular}

Significant at 5 percent level (Same letter in a given column did not differ significantly as per Duncan Multiple Range test) 
Table 2 : Effect on phenolic compounds on RGR and NAR in tomato under cold stress condition in Punjab during 2014-16

\begin{tabular}{|c|c|c|c|c|c|c|}
\hline \multirow[t]{2}{*}{ Treatments } & \multicolumn{3}{|c|}{$\begin{array}{l}\text { Relative growth rate } \\
\qquad\left(\mathrm{mg} \mathrm{g} \mathrm{day}^{-1}\right)\end{array}$} & \multicolumn{3}{|c|}{$\begin{array}{l}\text { Net assimilation rate } \\
\left(\mathrm{g} \text { leaf area } \mathrm{cm}^{2} \text { day }^{-1}\right)\end{array}$} \\
\hline & 30DAT & 60DAT & 90DAT & 30DAT & 60DAT & 90DAT \\
\hline \multicolumn{7}{|l|}{ Salicylic acid } \\
\hline $0.1 \mathrm{~m} \mathrm{M}$ & $274.36^{\mathrm{hi}}$ & $377.45^{\text {def }}$ & $469.51^{\mathrm{e}}$ & $0.046^{i j}$ & $0.23^{\text {gh }}$ & $1.98^{9}$ \\
\hline $0.5 \mathrm{~m} \mathrm{M}$ & $281.41^{\text {cdef }}$ & $385.01^{\mathrm{abcd}}$ & $475.48^{\text {cde }}$ & $0.054^{\text {fg }}$ & $0.28^{e f}$ & $2.26^{e}$ \\
\hline $1.0 \mathrm{~m} \mathrm{M}$ & $288.76^{\mathrm{ab}}$ & $391.79^{\mathrm{ab}}$ & $492.62^{\mathrm{ab}}$ & $0.063^{\mathrm{ab}}$ & $0.33^{\mathrm{ab}}$ & $3.74^{\mathrm{ab}}$ \\
\hline \multicolumn{7}{|c|}{ Methyl salicylic acid } \\
\hline $0.1 \mathrm{~m} \mathrm{M}$ & $270.70^{i}$ & $374.12^{f}$ & $467.12^{\mathrm{e}}$ & $0.043^{k}$ & $0.22^{\mathrm{h}}$ & $1.87^{9}$ \\
\hline $0.5 \mathrm{~m} \mathrm{M}$ & $277.99^{\text {fgh }}$ & $383.23^{\text {bcde }}$ & $473.47^{e}$ & $0.049^{\mathrm{hi}}$ & $0.27^{f}$ & $2.17^{\mathrm{ef}}$ \\
\hline $1.0 \mathrm{~m} \mathrm{M}$ & $283.80^{\text {bcde }}$ & $387.28^{\mathrm{abc}}$ & $483.36^{\text {bcd }}$ & $0.056^{\text {ef }}$ & $0.30^{\mathrm{de}}$ & $2.76^{d}$ \\
\hline \multicolumn{7}{|c|}{ Sulfo salicylic acid } \\
\hline $0.1 \mathrm{~m} \mathrm{M}$ & $274.39^{\mathrm{hi}}$ & $377.71^{\text {def }}$ & $469.31^{e}$ & $0.047^{\mathrm{j}}$ & $0.24^{\text {gh }}$ & $1.96^{9}$ \\
\hline $0.5 \mathrm{~m} \mathrm{M}$ & $281.24^{\text {def }}$ & $385.79^{\mathrm{abcd}}$ & $476.56^{\text {cde }}$ & $0.053^{\text {fg }}$ & $0.28^{\mathrm{ef}}$ & $2.34^{\mathrm{e}}$ \\
\hline $1.0 \mathrm{~m} \mathrm{M}$ & $287.34^{\mathrm{ab}}$ & $390.17^{\mathrm{ab}}$ & $490.50^{\mathrm{ab}}$ & $0.060^{\text {bcd }}$ & $0.32^{\mathrm{bcd}}$ & $3.31^{\mathrm{bc}}$ \\
\hline \multicolumn{7}{|l|}{ Benzoicacid } \\
\hline $0.1 \mathrm{~m} \mathrm{M}$ & $272.69^{i}$ & $376.02^{\text {ef }}$ & $468.12^{\mathrm{e}}$ & $0.045^{\mathrm{j} /}$ & $0.22^{\text {gh }}$ & $1.91^{9}$ \\
\hline $0.5 \mathrm{~m} \mathrm{M}$ & $279.70^{\text {efg }}$ & $384.16^{\mathrm{a}-\mathrm{e}}$ & $474.59^{\text {de }}$ & $0.051^{\text {gh }}$ & $0.27^{f}$ & $2.22^{\mathrm{e}}$ \\
\hline $1.0 \mathrm{~m} \mathrm{M}$ & $284.89^{\mathrm{abcd}}$ & $388.57^{\mathrm{ab}}$ & $484.73^{\mathrm{abc}}$ & $0.058^{\text {de }}$ & $0.31^{c d}$ & $2.88^{d}$ \\
\hline \multicolumn{7}{|c|}{ Acetyl salicylic acid } \\
\hline $0.1 \mathrm{~m} \mathrm{M}$ & $275.54^{\text {ghi }}$ & $379.26^{\text {cdef }}$ & $470.30^{\circ}$ & $0.047^{\mathrm{j}}$ & $0.24^{9}$ & $2.01^{\mathrm{fg}}$ \\
\hline $0.5 \mathrm{~m} \mathrm{M}$ & $287.82^{\mathrm{ab}}$ & $390.91^{\mathrm{ab}}$ & $491.67^{\mathrm{ab}}$ & $0.061^{a b c}$ & $0.32^{\mathrm{abc}}$ & $3.40^{\mathrm{ab}}$ \\
\hline $1.0 \mathrm{~m} \mathrm{M}$ & $286.25^{\mathrm{abc}}$ & $389.51^{\mathrm{ab}}$ & $489.10^{\mathrm{ab}}$ & $0.059^{\text {cde }}$ & $0.31^{\mathrm{bcd}}$ & $3.19^{c}$ \\
\hline Shelterbelt & $289.87^{\mathrm{a}}$ & $365.37^{a}$ & $493.65^{\mathrm{a}}$ & $0.064^{a}$ & $0.34^{\mathrm{a}}$ & $3.58^{a}$ \\
\hline Control (DW) & $264.20^{j}$ & $329.37^{9}$ & $451.46^{f}$ & $0.037^{\prime}$ & $0.18^{i}$ & $1.27^{\mathrm{h}}$ \\
\hline
\end{tabular}

Significant at 5 percent level (Same letter in a given column did not differ significantly as per Duncan Multiple Range test)

However, application of phenolic compounds significantly enhanced plant growth and led to increase in all biomarkers excluding specific leaf weight which decreased. The two year studies confirmed that foliar application of phenolic compounds significantly enhanced low temperature stress tolerance in tomato. Among all the phenolic compounds, salicylic acid (1.0 mM) was found to be more effective in protecting the plants against low temperature stress with enhanced growth and development of tomato plants as compared to non treated plants and this treatment was at par with shelterbelt. Similarly, Orabi et al. (2015) reported the enhanced effect of salicylic acid on endogenous growth regulators under low temperature, salicylic acid enhanced plant growth and cell division via regulation of other hormones (Zarghami et al., 2014) and mitigates abiotic stresses through increasing the growth regulating hormones (Shakirova et al., 2003). Similar results obtained by Sayyari et al. (2013) in watermelon showed that the growth parameters increased significantly with the application of salicylic acid under cold stress condition. Fahraji et al. (2014) reported enhanced photosynthetic activity with application of low concentration of salicylic acid, which enhanced their plant growth and development under low temperature stress.

The extent of water holding capacity in plant is measured by relative leaf water content (RLWC). The non-stressed plants did not display any symbolic contraction in RLWC despite they exhibited higher RLWC than stressed plants. The results of the present study revealed that low temperature stress caused reduction in RLWC of leaves $33.69,42.87$ and 49.99 in nontreated plants at 30,60 and $90 \mathrm{DAT}$, respectively, as compared to plants protected by shelterbelt $(69.95,83.12$ and 86.83$)$ (Table 3 ). Similarly, Slabbert and Kruger (2014) reported that RLWC significantly decreased with increasing stress. However, foliar application of phenolic compounds significantly enhanced RLWC in leaves at all stages during both the years. Among all the treated plants, salicylic acid (1.0 mM) observed maximum RLWC and enhanced it significantly as compared to non-treated plants at all the stages during study period. RLWC of cucumber presents a continuous decrease during low temperature stress and salicylic acid treatments induce RWC in leaf of stressed plants as compared to non treated plants (Ahmad et al., 2014; Iseri et al., 2013). Electrolyte leakage enables cell membrane injury to be assessed when plants are subjected to cold stress. Our studies revealed that low temperature stress adversely affects the membrane permeability measured as electrolyte leakage from leaf tissues in stressed plants at various growth stages (Table 3). The increase in membrane permeability was found as low temperature caused more electrolyte leakage from the membrane as compared to non stressed plants. Similarly, Khan et al. (2015) proved that the low temperature stress induces a 
Table 3 : Effect of phenolic compounds on relative leaf water content and electrolyte leakage in tomato under low temperature stress condition in Punjab during 2014-16

\begin{tabular}{|c|c|c|c|c|c|c|}
\hline \multirow[t]{2}{*}{ Treatments } & \multicolumn{3}{|c|}{ Relative leaf water content (\%) } & \multicolumn{3}{|c|}{ Electrolyte leakage (\%) } \\
\hline & 30DAT & 60DAT & 90DAT & 30DAT & 60DAT & 90DAT \\
\hline \multicolumn{7}{|l|}{ Salicylic acid } \\
\hline $0.1 \mathrm{~m} \mathrm{M}$ & $43.88^{\prime}$ & $63.33^{i}$ & $75.46^{\mathrm{e}}$ & $52.26^{\circ}$ & $40.90^{\circ}$ & $32.77^{\circ}$ \\
\hline $0.5 \mathrm{~m} \mathrm{M}$ & $56.00^{\mathrm{h}}$ & $73.27^{t}$ & $80.88^{d}$ & $48.31^{\mathrm{h}}$ & $34.82^{\mathrm{h}}$ & $27.23^{\mathrm{h}}$ \\
\hline $1.0 \mathrm{~m} \mathrm{M}$ & $68.04^{\mathrm{a}}$ & $83.09^{b}$ & $86.21^{\mathrm{ab}}$ & $36.61^{\mathrm{m}}$ & $29.32^{k}$ & $21.78^{n}$ \\
\hline \multicolumn{7}{|c|}{ Methyl salicylic acid } \\
\hline $0.1 \mathrm{~m} \mathrm{M}$ & $41.39^{\mathrm{m}}$ & $60.16^{\mathrm{j}}$ & $73.06^{\text {gh }}$ & $53.88^{\mathrm{b}}$ & $41.37^{\mathrm{b}}$ & $33.65^{\mathrm{b}}$ \\
\hline $0.5 \mathrm{~m} \mathrm{M}$ & $51.12^{j}$ & $70.23^{9}$ & $75.76^{e}$ & $50.63^{i}$ & $36.37^{9}$ & $29.85^{\circ}$ \\
\hline $1.0 \mathrm{~m} \mathrm{M}$ & $58.10^{9}$ & $76.13^{\mathrm{e}}$ & $80.69^{d}$ & $45.32^{j}$ & $32.20^{\circ}$ & $26.71^{i}$ \\
\hline \multicolumn{7}{|c|}{ Sulfo salicylic acid } \\
\hline $0.1 \mathrm{~m} \mathrm{M}$ & $44.48^{k}$ & $63.58^{i}$ & $74.73^{\text {ef }}$ & $53.16^{d}$ & $40.11^{d}$ & $31.85^{\mathrm{d}}$ \\
\hline $0.5 \mathrm{~m} \mathrm{M}$ & $55.20^{\mathrm{h}}$ & $72.80^{\circ}$ & $79.77^{d}$ & $49.24^{9}$ & $35.25^{9}$ & $28.21^{9}$ \\
\hline $1.0 \mathrm{~m} \mathrm{M}$ & $65.46^{d}$ & $79.37^{\circ}$ & $85.13^{b}$ & $41.03^{\prime}$ & $30.51^{\mathrm{jk}}$ & $24.39^{\prime}$ \\
\hline \multicolumn{7}{|l|}{ Benzoicacid } \\
\hline $0.1 \mathrm{~m} \mathrm{M}$ & $41.78^{\mathrm{m}}$ & $59.42^{\mathrm{j}}$ & $73.83^{\text {gh }}$ & $54.01^{\circ}$ & $41.66^{b}$ & $33.18^{\mathrm{c}}$ \\
\hline $0.5 \mathrm{~m} \mathrm{M}$ & $53.87^{i}$ & $67.93^{\mathrm{h}}$ & $76.87^{\circ}$ & $51.28^{\circ}$ & $36.89^{f}$ & $28.77^{f}$ \\
\hline $1.0 \mathrm{~m} \mathrm{M}$ & $60.05^{f}$ & $76.88^{\text {de }}$ & $80.61^{d}$ & $45.98^{i}$ & $32.11^{i}$ & $26.16^{\mathrm{j}}$ \\
\hline \multicolumn{7}{|c|}{ Acetyl salicylic acid } \\
\hline $0.1 \mathrm{~m} \mathrm{M}$ & $45.34^{k}$ & $63.80^{\circ}$ & $75.13^{\circ}$ & $50.70^{t}$ & $39.99^{\text {de }}$ & $32.06^{\mathrm{d}}$ \\
\hline $0.5 \mathrm{~m} \mathrm{M}$ & $66.96^{\mathrm{c}}$ & $81.40^{\mathrm{b}}$ & $85.96^{a}$ & $37.08^{\prime}$ & $29.77^{\mathrm{k}}$ & $23.25^{\mathrm{m}}$ \\
\hline $1.0 \mathrm{~m} \mathrm{M}$ & $63.82^{\mathrm{e}}$ & $77.46^{\mathrm{d}}$ & $82.73^{\circ}$ & $42.23^{k}$ & $31.47^{\mathrm{j}}$ & $25.38^{k}$ \\
\hline Shelterbelt & $69.95^{\mathrm{a}}$ & $83.12^{\mathrm{a}}$ & $86.83^{\mathrm{a}}$ & $34.51^{n}$ & $28.84^{k l}$ & $21.59^{\circ}$ \\
\hline Control (DW) & $33.69^{n}$ & $42.87^{\mathrm{k}}$ & $49.99^{\prime}$ & $65.64^{\mathrm{a}}$ & $55.08^{\mathrm{a}}$ & $46.87^{\mathrm{a}}$ \\
\hline
\end{tabular}

Significant at 5 percent level (Same letter in a given column did not differ significantly as per Duncan Multiple Range test)

significant increase in ion leakage through membranes in tomato. On the other hand, exogenous application of phenolic compounds help in overcoming the adverse effects of cold stress by restoring membrane permeability. Salicylic acid $(1.0 \mathrm{mM})$ was more effective in stabilizing the membranes in tomato plants growing under low temperature stress. Electrolyte leakage was greatly increased by cold stress and application of salicylic acid substantially reduced the electrolyte leakage (Lei et al., 2010; Ahmad et al., 2014). Salicylic acid facilitated maintenance of membrane functions through induction of antioxidant mechanism and elevated ion uptake, thereby protecting the plants against oxidative damage as reported by El-Tayed (2005).

Plant growth at low temperature resulted in lower number of flowers per cluster, number of fruits and yield per hectare as compared to plants protected by shelterbelt. Low temperature stress during vegetative growth stages significantly decreased the tomato yield (Table 4). The minimum number of flowers per cluster (4.12), number of fruits (22.62) and yield (224.34 q ha-1) were observed in non treated plants (Control), while the maximum number of flowers per cluster (6.77), number of fruits (38.24) and yield (586.50 q ha-1) were observed in non stressed plants (protected by shelterbelt) during study period. Plants grown at low temperature has low number of fruits and fruit yield per plant in tomato (Khan et al., 2015) which may be due to slow $\mathrm{CO}_{2}$ fixation and partitioning of photosynthates of fruit. However, significant increase in number of flowers per cluster, number of fruits and yield were observed with the foliar application of phenolics under stress. Among the phenolic compounds, salicylic acid $(1.0 \mathrm{~mm})$ was most effective as compared to other; it showed maximum flowers per cluster (6.75), number of fruits (38.04) and yield (579.41 $\mathrm{q}$ ha $^{-1}$ ) and was statistically at par with the plants protected by shelterbelt. Two year study confirmed that phenolic compounds protect plants against low temperature stress and enhanced production of tomato with increase in yield contribution attributes. Similarly, several workers have reported that the number of clusters and number of fruits and fruit yield were significantly increased with foliar application of salicylic acid in pepper (Hayat et al., 2010) and cucumber (Elvwan and Hamahyomy, 2009).

Low temperature stress during plant growth and development adversely affects quality of tomato fruit and reduces non-enzyme antioxidants such as lycopene, $\beta$-carotene and $\alpha$ tocopherol as per data shown in Table 4. Cold stress significantly reduced the lycopene content $\left(7.86 \mathrm{mg} 100 \mathrm{~g}^{-1}\right)$, $\beta$-carotene

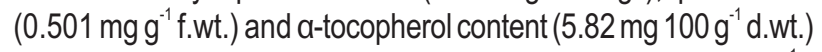

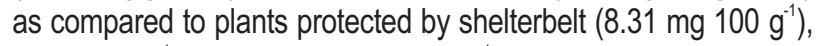
(0.630 $\mathrm{mg} \mathrm{g}^{-1}$ f.wt.) and (7.55 mg $100 \mathrm{~g}^{-1}$ d.wt.) respectively. Similar 
Table 4 : Effect of phenolic compounds on yield and quality attributes in tomato under low temperature stress condition in Punjab during 2014-16

\begin{tabular}{|c|c|c|c|c|c|c|}
\hline Treatments & $\begin{array}{l}\text { Number of flowers } \\
\text { per cluster }\end{array}$ & $\begin{array}{l}\text { Number of fruits } \\
\text { per plant }\end{array}$ & $\begin{array}{l}\text { Yield per } \\
\text { hectare (q) }\end{array}$ & $\begin{array}{l}\text { Lycopene } \\
\left(\mathrm{mg} \mathrm{100g}^{-1}\right)\end{array}$ & $\begin{array}{l}\beta \text {-carotene } \\
\text { ( } \mathrm{mg} \mathrm{g}^{-1} \text { f.wt.) }\end{array}$ & $\begin{array}{l}\text { a-tocopherol } \\
\text { (mg 100 g d.wt.) }\end{array}$ \\
\hline \multicolumn{7}{|l|}{ Salicylic acid } \\
\hline$(0.1 \mathrm{~m} \mathrm{M})$ & $5.90^{\text {ghi }}$ & $31.74^{e}$ & $448.98^{9}$ & $8.17^{\mathrm{hi}}$ & $0.573^{h}$ & $6.59^{\text {if }}$ \\
\hline$(0.5 \mathrm{~m} \mathrm{M})$ & $6.34^{\text {cde }}$ & $35.07^{\mathrm{cd}}$ & $513.31^{\text {de }}$ & $8.24^{\text {def }}$ & $0.590^{\text {ef }}$ & $7.02^{\mathrm{ab}}$ \\
\hline$(1.0 \mathrm{~m} \mathrm{M})$ & $6.75^{\mathrm{a}}$ & $38.04^{a}$ & $579.41^{\mathrm{ab}}$ & $8.30^{\mathrm{ab}}$ & $0.627^{\mathrm{ab}}$ & $7.54^{\mathrm{ab}}$ \\
\hline \multicolumn{7}{|c|}{ Methyl salicylic acid } \\
\hline$(0.1 \mathrm{~m} \mathrm{M})$ & $5.70^{\mathrm{fi}}$ & $31.19^{\circ}$ & $438.47^{9}$ & $8.12^{j}$ & $0.557^{j}$ & $6.32^{j}$ \\
\hline$(0.5 \mathrm{~m} \mathrm{M})$ & $6.17^{\text {efg }}$ & $34.45^{\mathrm{d}}$ & $499.24^{\mathrm{ef}}$ & $8.21^{9}$ & $0.582^{9}$ & $6.82^{g h}$ \\
\hline$(1.0 \mathrm{~m} \mathrm{M})$ & $6.41^{\text {bcde }}$ & $35.82^{\text {bcd }}$ & $532.14^{\text {cde }}$ & $8.26^{\text {de }}$ & $0.597^{\mathrm{de}}$ & $7.16^{\text {de }}$ \\
\hline \multicolumn{7}{|c|}{ Sulfo salicylic acid } \\
\hline$(0.1 \mathrm{~m} \mathrm{M})$ & $5.89^{g h i}$ & $31.74^{\mathrm{e}}$ & $451.00^{9}$ & $8.16^{\mathrm{hi}}$ & $0.565^{i}$ & $6.52^{i}$ \\
\hline$(0.5 \mathrm{~m} \mathrm{M})$ & $6.35^{\text {cde }}$ & $34.85^{c d}$ & $511.08^{\mathrm{de}}$ & $8.24^{\mathrm{ef}}$ & $0.594^{\mathrm{e}}$ & $7.11^{\text {ef }}$ \\
\hline$(1.0 \mathrm{~m} \mathrm{M})$ & $6.67^{\mathrm{ab}}$ & $37.31^{\mathrm{ab}}$ & $564.32^{\mathrm{abc}}$ & $8.29^{\mathrm{abc}}$ & $0.617^{b}$ & $7.44^{\mathrm{b}}$ \\
\hline \multicolumn{7}{|l|}{ Benzoicacid } \\
\hline$(0.1 \mathrm{~m} \mathrm{M})$ & $5.76^{\mathrm{hi}}$ & $31.32^{\mathrm{e}}$ & $442.85^{9}$ & $8.14^{i j}$ & $0.562^{\text {ig }}$ & $6.32^{j}$ \\
\hline$(0.5 \mathrm{~m} \mathrm{M})$ & $6.28^{\text {def }}$ & $34.26^{d}$ & $499.31^{\text {ef }}$ & $8.21^{\text {fg }}$ & $0.583^{\text {fg }}$ & $6.91^{9}$ \\
\hline$(1.0 \mathrm{~m} \mathrm{M})$ & $6.50^{\mathrm{abcd}}$ & $36.19^{\mathrm{abcd}}$ & $538.00^{\text {bcde }}$ & $8.26^{\text {cde }}$ & $0.602^{\text {cd }}$ & $7.26^{\text {cd }}$ \\
\hline \multicolumn{7}{|c|}{ Acetyl salicylic acid } \\
\hline$(0.1 \mathrm{~m} \mathrm{M})$ & $6.04^{\text {fgh }}$ & $31.92^{\mathrm{e}}$ & $461.14^{\mathrm{fg}}$ & $8.19^{g h}$ & $0.577^{\text {gh }}$ & $6.73^{\mathrm{h}}$ \\
\hline$(0.5 \mathrm{~m} \mathrm{M})$ & $6.71^{\mathrm{a}}$ & $37.93^{\mathrm{ab}}$ & $573.84^{\mathrm{abc}}$ & $8.30^{\mathrm{ab}}$ & $0.620^{b}$ & $7.47^{\mathrm{ab}}$ \\
\hline$(1.0 \mathrm{~m} \mathrm{M})$ & $6.61^{\mathrm{abc}}$ & $36.68^{\mathrm{abc}}$ & $549.46^{\text {abcd }}$ & $8.27^{\mathrm{bcd}}$ & $0.605^{\circ}$ & $7.33^{c}$ \\
\hline Shelterbelt & $6.77^{\mathrm{a}}$ & $38.24^{a}$ & $586.51^{a}$ & $8.31^{\mathrm{a}}$ & $0.629^{a}$ & $7.55^{\mathrm{a}}$ \\
\hline Control (DW) & $4.12^{j}$ & $22.62^{f}$ & $224.35^{\mathrm{h}}$ & $7.86^{k}$ & $0.502^{k}$ & $5.82^{k}$ \\
\hline
\end{tabular}

Significant at 5 percent level (Same letter in a given column did not differ significantly as per Duncan Multiple Range test)

results were obtained by Khan et al. (2015) who reported that low temperature stress significantly decreased lycopene and $\beta$ carotene in tomato. On the other hand, exogenous application of phenolic compounds significantly enhanced non-enzyme antioxidants. Among all the phenolic compounds, the maximum lycopene, $\beta$-carotene and a-tocopherol content were observed in plants treated with salicylic acid $(1.0 \mathrm{~mm})$ and it was statistically at par with plants protected by shelterbelt. Foliar application of salicylic acid significantly enhanced the lycopene content in tomato (Hafeznia et al., 2014). Huang et al. (2008) reported that pre-harvest treatment of salicylic acid could enhance lycopene and $\beta$-carotene content in tomato fruits during developmental stages and inhibit degradation of lycopene and $\beta$-carotene content during storage. This indicates that salicylic acid can activate lycopene biosynthesis pathway, including up-regulation of genes encoding the enzymes relating to lycopene level, during fruit development. The duration and temperature of exposure will determine if such an effect can occur, but tomato fruit when exposed to $32^{\circ} \mathrm{C}$ continuously will not turn red, remaining yellow even at full ripeness. Plants have the capacity to synthesize a lipophylic antioxidant known as a-tocopherol or Vitamin E. a-tocopherol scavenges free radicals in combination with other antioxidants (Massacci et al., 2008). Munne-Bosch and Algere (2003) reported that a-tocopherol helps in membrane stabilization and alleviates tolerance of plants during oxidative stress.
Tow year study revealed that application of salicylic acid and Acetyl salicylic acid mitigated cold stress by increasing the growth, yield and non-enzyme antioxidants in tomato fruits. It may thus be concluded that foliar application of Salicylic acid (1.0 mM) enhanced low temperature stress tolerance in tomato.

\section{References}

Ahmad, M. A., P.V. Murali and G. Marimuth: Impact of salicylic acid on growth, photosynthesis and compatible solute accumulation in Allium cepa L. subjected to drought stress. Int. J. Agri. Food. Sci., 4,22-30 (2014).

Asthir, B., K. Anjali and N. B. Singh: Thermodynamic behaviour of wallbound peroxidase from wheat leaves infected with stripe rust. Plant. Growth. Regul., 59,117-124 (2009).

El-Tayeb, M. A.: Response of barley grains to the interactive effect of salinity and salicylic acid. Plant Growth Regul., 45,215-224 (2005).

Elvwan, M. W. M. and M. A. M. Hamahyomy: Improved productivity and quality assocated with salicylic acid application in greenhouse pepper. Sci. Hortic., 122,521-526(2009).

Fahraji, S. S., M. A. Kheradmand, M. M. Raoofi and E. Fatahi: Effect of salicylic acid on germination, leaf area, shoot and root growth in crop plants. Int. Res. J. Appl. Basic. Sci., 8,1454-1458 (2014).

Hafeznia, M., K. Mashayekhi, G. Farshid and S. J. Mousavizadeh: Tomato morphological and biochemical characteristics in response to foliar applying of salicylic acid. Int. J. Biosci., 5, 237$243(2014)$. 
Hayat, Q., S. Hayat, M. Irfan and A. Ahmad: Effect of exogenous salicylic acid under changing environment : A review. Environ. Experi. Bot., 68,14-25(2010).

Hayat, S., Q. Hayat, M. N. Alyemeni, A. S. Wani, J. Pichtel and A. Ahmad: Role of proline under changing environments. Plant Signal Behav., 7,1456-1466 (2012).

Helyes, L., A. Lugasi, A. Pogonyi and Z. Pek: Effect of variety and grafting on lycopene content of tomato (Lycopersicon lycopersicum $\mathrm{L}$. karsten) fruit. Acta. Aliment., 38, 27-34 (2009).

Huang, J., M. M. Wang, Y. M. Bao, S. J. Sun, L. J. P. Zhang and H. S. Sriwrd: A novel WD40 protein subfamily regulated by salt stress in rice (Oryza sativa L.). Gene, 424, 71-79 (2008).

Iseri, O., D. A. Korpe, F. I. Sahin and M. Haberal: Hydrogen peroxide pretreatment of roots enhanced oxidative stress response of tomato under cold stress. Acta Physiol. Plant., 35,1905-1913 (2013).

Kaya, C., D. Higgs, F. Ince, B. M. Amador, A. Cukir and E Sakar: Ameliorative effect of potassium phosphate on salt stressed pepper and cucumber. J. Plant Nutr., 26, 807-820 (2003).

Khan, T. A., Q. Fariduddin and M. Yusuf: Lycopersicon esculentum under low temperature stress: An approach toward enhanced antioxidants and yield. Environ. Sci. Pollut. Res., DOI 10. 1007 / s11356-015-4658-5(2015).

Lei, T., H. Feng, X. Sun, Q.L. Dai, F. Zhang, H.G. Liang and H.H Lin: The alternative pathway in cucumber seedlings under low temperature stress was enhanced by salicylic acid. Plant Growth Regul., 60, 35-42 (2010).

Lukatkin, S., A. B. Bobinas and P. Duchovskis: Chilling injury in chillingsensitive plants : Areview. Agric., 99,111-124 (2012)

Massacci A., S. M. Nabiev Pietrosanti, S. K. Nematov, T. N. Chernikova, K. Thor and L. Leipner: Response of the photosynthetic apparatus of cotton (Gossypium hirsutum) to the onset of drought stress under field conditions studied by gas-exchange analysis and chlorophyll fluorescence imaging. Plant Physiol Biochem., 46, 189-95 (2008).

Munne-Bosch S. and L. Algere: Drought induced changes in the redox state of a -tocopherol, ascorbate and the diterpene carnosic acid in the chloroplasts of labiatae species differing in carnosic acid contents. Plant Physiol., 131, 1816-25 (2003).

Orabi, S. A., M. G. Dawood and S. R. Saleem: Comparative study between the physiological role of hydrogen peroxide and salicylic acid in alleviating low temperature stress on tomato plants grown under sand- ponic culture. Sci. Agric., 9,49-59 (2015).
Sadasivam, S. and A. Manickam: Biochemical Methods For Agri. Sci. pp 188-89. Wiley Eatern Ltd., New Delhi (1992).

Saltveit, M. E. and L. L. Morris: Overview on chilling injury of horticultural crops. In : Chilling injury of horticultural crops. (Ed. : C.Y. Wang) CRC Press., Boca Raton, pp. 3-15 (1990).

Sayyari, M., F. Ghanbari, S. Fatahi and F. Bavandpour: Chilling tolerance improving of watermelon seedling by salicylic acid seed and foliar application. Not. Sci. Biol., 1,67-73 (2013).

Shah, F., J. Huang, K. Cui, L. Nie, T. Shah, C. Chen and K. Wang: Impact of high-temperature stress on rice plant and its traits related to tolerance. J. Agri. Sci., 149, 545-556 (2011).

Shakirova, F. M., A. R. Sakhabutdinova, M. V. Bezrukova, R. A. Fatkhutdinova and D. R. Fatkhutdinova: Changes in the hormonal status of wheat seedlings induced by salicylic acid and salinity. Plant Sci., 164, 317-322 (2003).

Shi, Q., Z. Bao, Z. Zhu, Q. Ying and Q. Qian: Effect of different treatments of salicylic acid on heat tolerance, chlorophyll fluorescence and antioxidant enzyme activity in seedlings of Cucumis sativa L. Plant Growth Regul., 48,127-135 (2006).

Shi, X. M., F. Jin, Y. T. Huang, X. W. Du, C. M. Li, M. Wang, H. Shao, M. J. Jin and J. Wang: Simultaneous determination of five plant growth regulators in fruit by modified quick, easy, cheap, effective, rugged and safe (QuEChERS) extraction and liquid chromatographytandem mass spectrometry. J. Agric. Food. Chem., 60, 60-65 (2012).

Slabbert, M. M. and G.H.J. Krüger: Antioxidant enzyme activity, proline accumulation, leaf area and cell membrane stability in water stressed Amaranthus leaves. South African J. Bot., 95,123-128 (2014).

Strauss, A. J., G. H. J. Kruger, R. J. Strasser and P. D. R. Heerden: The role of lowsoil temperature in the inhibition of growth and PSII function during dark chilling in soybean genotypes of contrasting tolerance. Physiol. Plant., 131, 89-105 (2007).

Watanabe, K., T. Saito, S. Hirota, B. Takahashi and N. Fujishita: Carotenoid-pigments in orange, light orange, green and white flesh colored fruits of melon (Cucumis melo L). J. Jpn. Soc. Food Sci., 38,153-159(1991).

Zarghami, M., M. Shoor, A. Ganjali, N. Moshtaghi and A. Tehranifar: Effect of salicylic acid on morphological and ornamental characteristics of Petunia hybrida at drought stress. Indian J. Fund. Appl. Life Sci., 4, 523-532(2014).

Zhang, Y., M. Zhang and H. Yang: Postharvest chitosan-g-salicylic acid application alleviates chilling injury and preserves cucumber fruit quality during cold storage. Food Chemistry, 174, 558-563 (2015). 